\title{
Answer to a Quiz : Pacemaker on page ... and case discussion
}

\section{Answer: 3}

The beginning of the tracing indicates a DDD mode programming with both atrial and ventricular pacing which seem efficient at $60 \mathrm{bpm}$ (with best visible atrial activity seen after the first beat), and during six beats. The 7th spike is an atrial one, not followed by a ventricular activity, neither paced nor spontaneous. The last three beats of the tracing now represent atrial spikes followed by spontaneous QRS complexes, suggesting the presence of an intrinsic ventricular activity. This behaviour is more suggestive of an AAl mode programming.

One can note that the QRS morphology is a RBBB paced QRS suggesting a possible left ventricular origin for the ventricular lead. The course of the ventricular lead through a permeable foramen ovale was excluded by the post-operative chest X-ray. Finally this RBBB morphology may be explained by the position of the recording electrode on the chest during the surface-ECG.

$1 /$ It is then impossible to confirm the presence of an intermittent ventricular pacing dysfunction as one would expect to see a ventricular spike not followed by a paced ventricular complex.

2/ The paced atrial activity is difficult to see on the tracing, and may be best visible after the first spike. This is often the case, and requires an amplification on the ECG recording to better visualize them. One can argue about the absence of efficient capture after the 6th atrial spike, but a spontaneous atrial activity seems visible before the spike, and may explain that the atria are now in an absolute refractory period.

\section{3/ There is no dysfunction.}

The tracing is a normal recording of a switching mode from a DDD to an AAI mode. The SafeR algorithm has been designed to avoid any useless and deleterious ventricular pacing.

The pacemaker is programmed to switch from an AAI to a DDD mode in case of : 2 consecutive blocked $P$ waves; 3 blocked P waves out of 12 beats; 7 long PR intervals.

This is a unique tracing of switching mode from a DDD programming to an AAl programming with normal AV conduction and spontaneous QRS complexes, which allows a single blocked $P$ wave (the 7th complex). This switching occurs every hundred beat, or every morning at 8 AM.

4/ The absence of ventricular spike after an atrial spike, may be due to an inhibition on the ventricular lead. However, a protection system exists to avoid this inhibition. It is defined as the presence of a ventricular safety window which would then occur $100 \mathrm{mec}$ after the atrial pacing spike. This is not the case here.

Sok-Sithikun Bun, Karim Hasni Cardiology Department, Princess Grace Hospital, Monaco

Peer-review: internal Conflict of interest: None to declare Authorship: S.S.B. and K.H. equally contributed to preparation of quiz Acknowledgement and funding: None to declare

Address for Correspondence: Sok-Sithikun Bun, Cardiology Department, Princess Grace Hospital, Monaco Email : sithi.bun@gmail.com Phone : +377979897 71 - Fax : + 37797989732

Received: 23.03.2019 Accepted: 24.03.2019 